\title{
Erratum to: Decreased Tongue Pressure is Associated with Sarcopenia and Sarcopenic Dysphagia in the Elderly
}

\author{
Keisuke Maeda · Junji Akagi
}

Published online: 8 November 2014

(C) Springer Science+Business Media New York 2014

\section{Erratum to: Dysphagia}

DOI 10.1007/s00455-014-9577-y

In the original publication of the article the percentages of sarcopenia in Table 4 were interchanged and published incorrectly.

The authors apologize for this error and the correct version of Table 4 is given below.
The online version of the original article can be found under doi:10.1007/s00455-014-9577-y.

K. Maeda $(\bowtie)$

Department of Internal Medicine, Tamana Regional Health

Medical Center, 2172 Tamana, Taman City, Kumamoto

Prefecture 865-0005, Japan

e-mail: maeda4work@tng.bbiq.jp

J. Akagi

Department of Surgery, Tamana Regional Health Medical

Center, Tamana, Japan
Table 4 Univariate analyses show differences in parameters between the non-dysphagia group and dysphagia group

\begin{tabular}{lllr}
\hline Variable & $\begin{array}{l}\text { Dysphagia } \\
(n=44)\end{array}$ & $\begin{array}{l}\text { Non-dysphagia } \\
(n=60)\end{array}$ & $p$ value \\
\hline MTP (kPa) & $14.7 \pm 8.0$ & $25.3 \pm 7.7$ & $<0.001$ \\
Age (years) & $84.8 \pm 4.1$ & $84.2 \pm 5.6$ & 0.502 \\
AC (cm) & $20.8 \pm 3.1$ & $25.3 \pm 3.3$ & $<0.001$ \\
TSFc (mm) & $8.3 \pm 5.2$ & $13.5 \pm 7.1$ & $<0.001$ \\
AMAd (cm $\left.{ }^{2}\right)$ & $26.7 \pm 6.8$ & $35.7 \pm 8.0$ & $<0.001$ \\
BMIe (kg/m $\left.{ }^{2}\right)$ & $18.4 \pm 3.6$ & $21.8 \pm 4.0$ & $<0.001$ \\
Albumin (g/dL) & $2.8 \pm 0.6$ & $3.4 \pm 0.6$ & $<0.001$ \\
MNA-SF (points) & $7.1 \pm 2.9$ & $9.9 \pm 2.8$ & $<0.001$ \\
CONUT (points) & $5.8 \pm 2.9$ & $3.8 \pm 2.7$ & $<0.001$ \\
Barthel index (points) & $36.8 \pm 34.1$ & $56.4 \pm 38.9$ & 0.015 \\
Sex (men, \%) & 31.8 & 36.7 & 0.679 \\
Dentures (\%) & 59.1 & 60.0 & 1.000 \\
Sarcopenia (\%) & 31.8 & 1.7 & $<0.001$ \\
\hline
\end{tabular}

$M T P$ maximum voluntary tongue pressure against the palate, $A C$ midupper arm circumference, $T S F$ triceps skinfold thickness, $A M A$ arm muscle area, $B M I$ body mass index, MNA-SF Mini-Nutritional Assessment short form 